\title{
Uma Sistematização dos Estudos Nacionais em Psicologia Ambiental
}

Una Sistematización de los Estudios Nacionales en Psicología Ambiental A Systematization of the National Studies in Environmental Psychology

\author{
Maísa Hodecker \\ ORCID: http://orcid.org/0000-0001-5273-1575 \\ Universidade Federal de Santa Catarina, Santa Catarina/Brasil \\ Igor Schütz dos Santos \\ ORCID: http://orcid.org/0000-0001-9000-4934 \\ Universidade Federal de Santa Catarina, Santa Catarina /Brasil \\ Maíra Longhinotti Felippe \\ ORCID: http://orcid.org/0000-0002-0392-7480 \\ Universidade Federal de Santa Catarina, Santa Catarina/Brasil
}

Daniela Ribeiro Schneider

ORCID: http://orcid.org/0000-0002-2936-6503 Universidade de Valência, Valência/ Espanha

Declaração de Direito Autoral

A submissão de originais para este periódico implica na transferência, pelos autores, dos direitos de publicação impressa e digital. Os direitos autorais para os artigos publicados são do autor, com direitos do periódico sobre a primeira publicação. Os autores somente poderão utilizar os mesmos resultados em outras publicações indicando claramente este periódico como o meio da publicação original. Em virtude de sermos um periódico de acesso aberto, permite-se o uso gratuito dos artigos em aplicações educacionais e científicas desde que citada a fonte conforme a licença CC-BY da Creative Commons.

Ariane Kuhnen

ORCID: http://orcid.org/0000-0001-9635-9306 Universidade Federal de Santa Catarina, Santa Catarina /Brasil

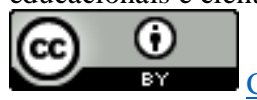

Creative Commons Atribuição 4.0 Internacional.

\section{Resumo}

A Psicologia Ambiental (PA) é uma disciplina que se propõe a estudar a inter-relação entre pessoaambiente, a partir da perspectiva de que ambos estão inseridos em um sistema de mútua influência. Com o objetivo de apresentar uma sistematização de dados dos estudos em Psicologia Ambiental no contexto nacional, este estudo realizou uma análise da produção científica de artigos relacionados com o tema, disponíveis nas bases de dados Scielo, Pepsic, Indexpsi, BVS, LILACS. Utilizou-se os descritores pessoa, ambiente e psicologia ambiental, selecionados segundo os Descritores em Ciências da Saúde (DeCS). A partir da amostra nacional de 29 artigos científicos, foi verificado que a relação pessoa-ambiente em suas origens adotava uma perspectiva individualista, focada nos efeitos psicológicos que o ambiente causava nas pessoas. Posteriormente, estudou-se a pessoaambiente em uma perspectiva interacionista, na qual ambos são unidades separadas e possuem uma relação de causa e efeito. Já a partir de uma visão mais atual, pessoa e ambiente são vistos inseridos em um sistema de inter-relação, em que ambos se definem e se modificam mutuamente. Foi possível identificar que a ontologia atual é materialista, a antropologia é transacionalista e agente e a epistemologia é interacionista e construtivista. Verificou-se que há duas características principais na PA: construção do conhecimento científico a partir da abordagem multimétodos e interdisciplinar. 
Sugere-se a futuras pesquisas incorporar questões culturais nas investigações pessoa-ambiente, devido ao modo como influencia visões de mundo e construções urbanas e privadas.

Palavras-chave: Pessoa; Ambiente; Epistemologia; Psicologia Ambiental.

\title{
Resumen
}

La Psicología Ambiental (PA) es una disciplina que se propone a estudiar la interrelación entre persona-ambiente, desde la perspectiva de que ambos están insertados en un sistema de mutua influencia. Con el objetivo de presentar una sistematización de datos de los estudios en Psicología Ambiental en el contexto nacional, este estudio realizó un análisis de la producción científica de artículos relacionados con el tema, disponibles en las bases de datos Scielo, Pepsic, Indexpsi, BVS, LILACS. Se utilizó los descriptores persona, ambiente y psicología ambiental, seleccionados según los Descriptores en Ciencias de la Salud (DeCS). A partir de la muestra nacional de 29 artículos científicos, se verificó que la relación persona-ambiente en sus orígenes adoptaba una perspectiva individualista, enfocada en los efectos psicológicos que el ambiente causaba en las personas. Posteriormente, se estudió la persona-ambiente en una perspectiva interaccional, en la cual ambos son unidades separadas y poseen una relación de causa y efecto. A partir de una visión más actual, la persona y el ambiente se ven insertados en un sistema de interrelación, en el que ambos se definen y se modifican mutuamente. Fue posible identificar que la ontología actual es materialista, la antropología es transacionalista y agente y la epistemología es interaccionista y constructivista. Se verificó que hay dos características principales en la PA: construcción del conocimiento científico a partir del abordaje multiforme e interdisciplinario. Se sugiere a futuras investigaciones incorporar cuestiones culturales en las investigaciones persona-ambiente, debido al modo como influencia visiones de mundo y construcciones urbanas y privadas.

Palabras clave: Persona; Ambiente; Epistemología; Psicología Ambiental.

\begin{abstract}
Environmental Psychology (PA) is a discipline that studies the interrelation between personenvironment, from the perspective that both are inserted in a system of mutual influence. With the objective of presenting a data systematization of studies in Environmental Psychology in the national context, this study carried out an analysis of the scientific production of articles related to the theme, available in SciELO, Pepsic, Indexpsi, VHL, LILACS databases. We used the descriptors person, environment and environmental psychology, selected according to the Descriptors in Health Sciences (DeCS). From the national sample of 29 scientific articles, it was verified that the personenvironment relationship in its origins adopted an individualistic perspective, focused on the psychological effects that the environment caused in the people. Subsequently, the personenvironment was studied in an interactionist perspective, in which both are separate units and have a cause-and-effect relationship. From a more current view, the person and environment are seen inserted in an interrelated system, in which both define and modify each other. It was possible to identify that the current ontology is materialist, anthropology is transactionalist and agent and epistemology is interactionist and constructivist. It was verified that there are two main characteristics in the PA: construction of scientific knowledge from the multi-method and interdisciplinary approach. For future researches is suggested to incorporate cultural issues into human-environment investigations, owing to the way it influences worldviews and urban and private constructions.
\end{abstract}

Keywords: Person; Environment; Epistemology; Environmental Psychology. 


\section{Introdução}

De modo amplo, a Psicologia busca compreender o indivíduo em suas dimensões psicológicas, comportamentais, cognitivas, levando em conta sua subjetividade. Embora seja uma área abrangente composta por diferentes correntes teóricas que buscam analisar os fenômenos psicológicos de acordo com seus vieses particulares, os fenômenos físicos intrincados na relação pessoa-ambiente são, por vezes, desconsiderados ou anulados completamente. Justamente nesse quesito está o diferencial da Psicologia Ambiental (PA): considerar o indivíduo imerso a todo momento em um ambiente físico que o influencia assim como é influenciado por ele, em um processo que se retroalimenta. Compreende-se que ambiente e pessoa não são unidades isoladas, mas partes de um mesmo sistema, em que se definem e se transformam mutuamente. Para compreender melhor essa disciplina, a seguir será apresentada uma breve descrição do seu nascimento e principais corresponsáveis pela sua expansão (Elali, 1997; Albuquerque \& Albuquerque, 2005; Machado, Schubert, Albuquerque, \& Kuhnen, 2016).

A Psicologia Ambiental emerge como área da psicologia responsável por resolver problemas oriundos da interação ambientepessoa. Pol (1993) descreve em seu livro "Psicologia Ambiental na Europa: da Psicologia Arquitetural à Psicologia Ambiental Verde" (Environmental Psychology in Europe: from Architectural Psychology to Green Psychology) uma retrospectiva histórica da PA, desde meados de 1980 até a década de 90 na Europa. Nos fins dos anos 1950 e início dos anos 60, arquitetos que usualmente trabalhavam para seus clientes privados, foram incumbidos da tarefa de planejar um grande número de habitações a população desabrigada no período pós-guerra. Os arquitetos tinham que planejar uma quantidade significativa moradias, responder às expectativas estéticas de usuários, além de lidar com uma diversidade de perfis de usuários e atentar-se ao contexto sociocultural de cada um (Tassara \& Rabinovich, 2003).
Ao executar programas habitacionais em larga escala com a particularidade de residir indivíduos que foram mutilados, fragilizados e traumatizados pela guerra, os arquitetos uniram-se a cientistas do comportamento, e ocorreu a consciência que o ambiente construído deveria ir além de princípios de construção e estética, mas acoplar as necessidades psicológicas e comportamentais dos seus futuros ocupantes. Havia uma nova demanda que deveria ser considerada para além dos princípios básicos da arquitetura do ambiente. Assim, ocorre o surgimento da Psicologia Arquitetural. Destaca-se que alguns construtos foram essenciais à compreensão e ação da criação dessa disciplina: Privacidade, Territorialidade e Apropriação (Rivlin, 2003).

Após este período, houve a expansão da Psicologia Arquitetural a partir de conflitos não somente de campo-cidade, mas entre terceiro e primeiro mundos, países ricos e pobres, com diferenças políticas e sociais explícitas (Pol, 1993). Aproximadamente em 1990, surge a Psicologia Verde, ou também chamada de Psicologia Ambiental Verde (Pol, 2006; 2007). Em setembro de 1995, o Journal of Environmental Psychology publica uma edição especial de sua revista dedicado a estudos que contemplassem a Psicologia Verde. Nesse sentido, a revista contemplou artigos com temáticas relacionadas ao manejo de recursos naturais renováveis e nãorenováveis, conservação de energia, comportamento ecologicamente responsável e sustentabilidade. Tanto a Psicologia Arquitetural quanto a Psicologia Verde são tendências atuais no âmbito da PA (Moser, 1998).

$\mathrm{O}$ primeiro autor a defender que os psicólogos deveriam estudar a representatividade do design devido aos estímulos ambientais que são transmitidos via design foi Brunswik em 1943. Outro influente pesquisador à PA foi Kurt Lewin, ao introduzir a perspectiva de considerar a dimensão ambiental na Psicologia. Lewin contribuiu principalmente com a criação da Teoria de Campo, considerando o ambiente 
físico nas pesquisas, e a pesquisa-ação, demonstrando a importância da pesquisa científica associada às mudanças sociais concretas. A partir da década de 70 a PA se firma como disciplina científica, englobando em sua proposta o contexto das relações entre pessoa-ambiente, ambientes físicos e problemas ambientais, na busca de atuações pautadas na resolução de problemáticas e produção de conhecimento científico para a ampliação desse saber (Pol, 1993; Valera, 1996).

Desde então, em Psicologia Ambiental os ambientes são compreendidos como únicos e dotados de qualidades que dependem fortemente do percebedor e sua subjetividade. Ambientes naturais ou construídos possuem capacidade de proporcionar sentimentos de valência positiva, bem-estar e qualidade de vida, por exemplo, assim como o inverso, sentimentos de valência negativa, como estresse e desconforto. Dessa forma, a Psicologia Ambiental não somente investiga o indivíduo em um ambiente físico, como aspectos psicológicos e físicos envolvidos nessa relação. Diante de tal problemática, comumente adota-se uma abordagem multimétodos para buscar investigar todos os fenômenos envolvidos nessa relação (CorralVerdugo, 2005; Elali, 2007).

O uso da abordagem multimétodos é justificado principalmente devido a díade pessoa-ambiente, sendo necessários métodos direcionados aos dois objetos de investigação. Assim, Psicologia Ambiental estuda a pessoa e seu entorno, sempre inserida em um ambiente físico e social em inter-relação. O entorno está cercado por dimensões físicas e sociais que influenciam diretamente a percepção, avaliação e atitudes do indivíduo frente a esse contexto. Compreende-se que o modo como cada indivíduo irá perceber e interpretar cada ambiente dependerá ainda de certas características, experiências e valores pessoais, tornando o modo de ver o mundo único. A inter-relação e seu estudo pessoa-ambiente investiga os efeitos de ambientes físicos sobre o ser humano, não como um sujeito passivo, mas agente ativo, porém constantemente sendo influenciado pelo entorno. Ressalta-se que o entorno também está passível de sofrer influências de seus usuários. Desse modo, o foco das pesquisas em PA pode ser centrado no ambiente físico, na pessoa ou na relação entre ambos. Porém, em qualquer pesquisa, considera-se a premissa central de que um causa efeito sobre o outro, ambos são ativos e fazem parte de um mesmo sistema transacional (Tuan, 1983; Machado et al., 2016).

Como ambos os objetos se transformam mutuamente, considera-se que ultrapassa uma relação, pois há dois agentes ativos que atuam um sobre o outro, ou seja, uma inter-relação dinâmica. Toda pessoa está imersa a todo instante em ambientes, sejam eles construídos e planejados ou naturais, recebendo estímulos e mensagens que podem comunicar tanto sentimentos de valência positiva, como negativa. Afirma-se, portanto, que não se deve isolar e estudar ou um ou outro per se, pois ambos possuem o mesmo peso. Por fim, o diferencial e especificidade da PA diz respeito a forma de analisar e considerar o modo como o indivíduo avalia e percebe o ambiente e como está sendo influenciado por ele (Wiesenfeld, 2005).

Além desse diferencial, a PA a busca promover espaços mais harmônicos $\mathrm{e}$ confortáveis, sustentabilidade ambiental e bem-estar humano por meio do estudo do ambiente circundante (Vlek, 2003). Tendo em vista tais objetivos, faz-se necessário adotar algumas ações durante o estudo nesse campo: 1) a relação pessoa-ambiente no contexto natural, sendo analisada em sua totalidade; 2) abordar a relação pessoa-ambiente de forma holística; 3) incorporar diversas perspectivas teóricas para tentar explicar o fenômeno estudado; 4) ressaltar o social aliado à relação homem e ambiente; 5) abordar o fenômeno de forma multiprofissional e interdisciplinar; 6) buscar a aplicação do conhecimento em PA para melhorar a qualidade do ambiente, e, consequentemente, à saúde de seus usuários (Pinheiro, 1997; Günther, Elali, \& Pinheiro, 2008; Felippe, 2009). 
Como dito, busca-se aliar diversas perspectivas teóricas e disciplinas para explicar os fenômenos pessoa e ambiente. Portanto, espera-se uma atuação multiprofissional e interdisciplinar, utilizando de várias profissões e que conversem entre si para complementar os estudos ligados a pessoa e ambiente. Como exemplo, a Biologia e a Geografia podem ser associadas no estudo com a Psicologia para estudar o ambiente natural, assim como a Arquitetura, Urbanismo, Ergonomia e Engenharias para estudar ambientes construídos e planejados (Pinheiro, 1997). Estes foram apenas alguns exemplos de disciplinas que se propunham a utilizar em seus estudos a Psicologia Ambiental para explicar, investigar e analisar seus fenômenos, sejam eles mais centrados no ambiente físico ou na pessoa em suas dimensões psicológicas. Nesse sentido, o ideal é a integração de diferentes disciplinas para obter uma visão mais unificada sobre o objeto de estudo (Ittelson, Proshansky, Rivlin, \& Winkel, 2005; Kruse, 2005).

Particularmente, a proposta deste trabalho foi apresentar uma sistematização da literatura acerca dos estudos em Psicologia Ambiental, de modo a identificar o objeto de estudo, aspectos ontológicos, epistemológicos e antropológicos, além dos métodos e metodologias mais empregados em PA. Para tanto, realizou-se uma análise da produção científica de artigos, disponíveis nas bases de dados Scielo, Pepsic, Indexpsi, BVS e LILACS.

\section{Método}

Esta pesquisa foi construída por meio de revisão integrativa de literatura. De acordo com Beyea e Nicoll (1998), a revisão integrativa corresponde a um tipo de pesquisa que sumariza e extrai conclusões globais de um corpo de literatura a partir de um tema central. Para tanto, adotou-se as seguintes etapas de pesquisa: 1) seleção da questão temática; 2) estabelecimento dos critérios de inclusão e exclusão; 3) análise e interpretação dos dados; e 4) apresentação da revisão integrativa. Quanto ao tipo de pesquisa, caracteriza-se como artigo de análise. Marconi e Lakatos (2017) sustentam que esse tipo de artigo científico engloba uma descrição do assunto, classificação e definição do assunto, levando em consideração a estrutura, a forma, os objetivos propostos e a finalidade do tema, assim como apresenta detalhes e exemplos.

Nas bases de dados Scielo, Pepsic, Indexpsi, BVS, LILACS utilizou-se os descritores "pessoa", "ambiente" e "psicologia ambiental", selecionados segundo os Descritores em Ciências da Saúde (DeCS), utilizando o cruzamento dos descritores por meio do operador booleano "and". Os critérios de inclusão foram de artigos empíricos e teóricos, publicados nas referidas bases de dados, com acesso livre, desde que fossem artigos completos. Nos achados, buscou-se identificar a epistemologia (modo de fazer conhecimento), ontologia (o estudo do ser) e antropologia (modo de conceber o homem) da PA por meio do referencial teórico. Não foram estabelecidos anos de refinamento, pois como a PA é uma disciplina desenvolvida recentemente, poucos artigos seriam contemplados. Destaca-se que a pesquisa englobou estudos publicados até maio de 2018, quando ocorreu a coleta de dados. Foram selecionados apenas artigos em português realizados no Brasil. Salienta-se que a pesquisa foi realizada por um dos pesquisadores, e posteriormente foi submetida a revisão por pares. Foi elaborado um roteiro de pesquisa, contendo os critérios de inclusão e exclusão supracitados, e cada pesquisador analisou as produções nas bases de dados, reproduzindo a pesquisa de forma similar. Além dos autores obtidos pela busca nas bases de dados, durante a discussão e resultados foram realizadas inferências com outros autores que também estudam a Psicologia Ambiental e estudos pessoa-ambiente.

\section{Resultados}

Nas bases de dados, foram obtidos 617 resultados a partir dos descritores supracitados (Scielo: 16, Pepsic: 3, Indexpsi: 6, BVS: 561, LILACS: 31). Foram filtrados a priori os artigos científicos com acesso livre e completo 
em português. Os 16 artigos encontrados na base Scielo foram todos incluídos na análise. $\mathrm{Na}$ base Pepsic um artigo foi incluso e dois foram excluídos por duplicação. $\mathrm{Na}$ base Indexpsi, um artigo foi incluído devido a congruência com os critérios de inclusão, quatro foram excluídos por duplicação e um excluído por ser outra modalidade de trabalho científico (resumo expandido). Na BVS foram incluídos nove artigos científicos, 211 foram excluídos por não conterem texto completo disponível, 330 por serem estudos realizados fora do Brasil, 11 foram excluídos por duplicação e um artigo foi excluído por estar em língua estrangeira. Na base LILACS, dois artigos científicos foram incluídos na análise, 20 artigos foram excluídos por duplicação, sete artigos estavam incongruentes com a temática, um artigo excluído por estar em língua estrangeira e um deles compreendida a resumo expandido. Portanto, ao final da filtragem e aplicação dos critérios de inclusão e exclusão, a amostra consistiu em 29 artigos científicos das bases nacionais. $\mathrm{O}$ procedimento de busca de artigos adotado nesta pesquisa pode ser identificado na Figura 1.

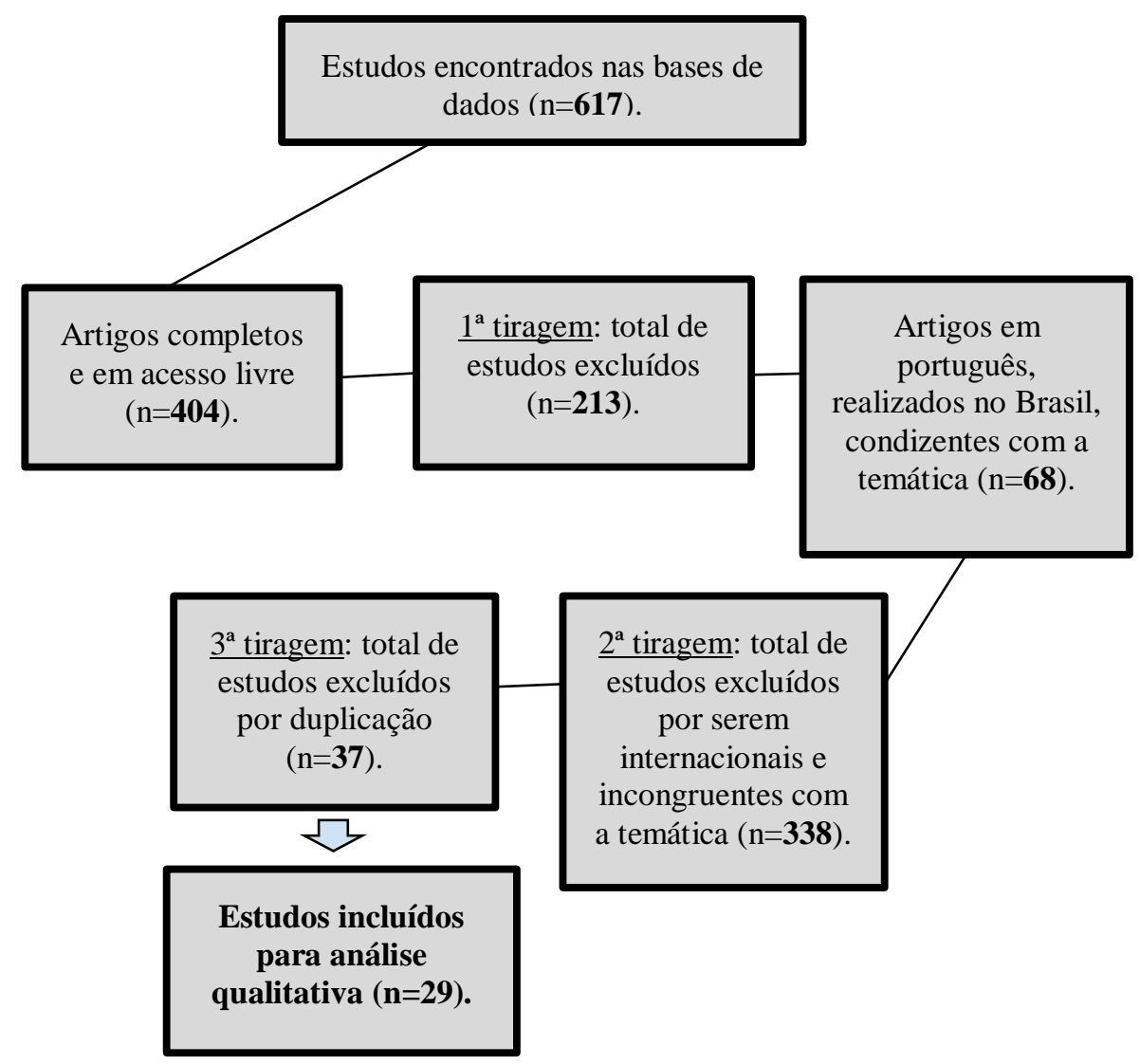

Figura 1. Fluxograma da pesquisa.

\section{Discussão \\ A investigação científica em sua dimensão metodológica e temática}

De modo geral, analisou-se que a abordagem multimétodos é citada como forte característica no que cerne a investigação pessoa-ambiente (Elali, 1997; Pinheiro, 1997; Moser, 1998), mas apenas nove artigos utilizaram deste método de pesquisa (Elali,
2003; Khoury \& Günther, 2008; Salvador, Florindo, Reis, \& Costa, 2009; Ros \& Cruz, 2011; Luz \& Kuhnen, 2013; Martinez \& Emmel, 2013; Porciuncula \& Porto, 2014; Machado et al., 2016; Albuquerque, Kuhnen, \& Silva, 2016). A maioria dos achados (15) corresponde a estudos teóricos (Carneiro \& Bindé, 1997; Elali, 1997; Pinheiro 1997; Moser, 1998; Rivlin, 2003; Günther, 2003; Campos-de-Carvalho, 2003; Vlek, 2003; 
Alencar \& Freire, 2007; Lima \& Bomfim, 2009; Kuhnen, Felippe, Luft, \& Faria, 2010; Polli \& Kuhnen, 2011; Felippe \& Kuhnen, 2012; Lopez, Felippe, \& Kuhnen, 2012; Corrêa \& Bassani, 2015) e cinco estudos de levantamento de dados (Macedo, Oliveira, Günther, Alves, \& Nóbrega, 2008; Farias \&
Pinheiro, 2013; Polli \& Camargo, 2013; Araújo \& Santos, 2014; Zuchiwschi \& Fantini, 2015). O Quadro 1, exposto a seguir, resume os resultados mais expressivos da seleção de trabalhos relacionados à $\mathrm{PA}$ no que diz respeito aos métodos, às técnicas e ao contexto de investigação (natural ou artificial).

Quadro 1. Sistematização dos dados dos estudos contemplados na pesquisa

\begin{tabular}{|c|c|c|c|c|}
\hline$n^{\mathbf{o}}$ & $\begin{array}{c}\text { Autor e ano } \\
\text { de publicação }\end{array}$ & Objetivo da pesquisa & $\begin{array}{l}\text { Método de } \\
\text { pesquisa* }\end{array}$ & Técnicas e instrumentos \\
\hline 1 & Elali (2003) & $\begin{array}{l}\text { Investigar o ambiente físico de instituições } \\
\text { de educação infantil. }\end{array}$ & Multimétodos & $\begin{array}{c}\text { Descrição de elementos; } \\
\text { observação } \\
\text { comportamental; } \\
\text { abordagem direta aos } \\
\text { usuários (entrevista, } \\
\text { questionários, desenho } \\
\text { temático). }\end{array}$ \\
\hline 2 & $\begin{array}{c}\text { Carneiro e } \\
\text { Bindé (1997) }\end{array}$ & $\begin{array}{l}\text { Discutir a relação entre Psicologia Ecológica } \\
\text { e contextos diários. }\end{array}$ & Estudo teórico & \\
\hline 3 & $\begin{array}{l}\text { Albuquerque } \\
\text { et al. (2016) }\end{array}$ & $\begin{array}{l}\text { Identificar lugares de descanso preferidos } \\
\text { por estudantes em um campi universitário }\end{array}$ & Multimétodos & $\begin{array}{l}\text { Entrevista associada ao } \\
\text { ambiente fotografado. }\end{array}$ \\
\hline 4 & Rivlin (2003) & $\begin{array}{c}\text { Examinar pressupostos tradicionais e } \\
\text { contemporâneos da PA. }\end{array}$ & Estudo teórico & \\
\hline 5 & $\begin{array}{l}\text { Günther } \\
\text { (2003) }\end{array}$ & $\begin{array}{l}\text { Discutir os conceitos de espaço pessoal, } \\
\text { territorialidade, apinhamento e privacidade e } \\
\text { sua relação com a mobilidade. }\end{array}$ & Estudo teórico & \\
\hline 6 & $\begin{array}{l}\text { Campos-de- } \\
\text { Carvalho } \\
\text { (2003) }\end{array}$ & $\begin{array}{c}\text { Caracterizar pesquisas ecológicas e } \\
\text { diferenciá-las de pesquisas não ecológicas. }\end{array}$ & Estudo teórico & \\
\hline 7 & Vlek (2003) & $\begin{array}{c}\text { Apresentar uma visão da ciência do } \\
\text { comportamento sobre o desenvolvimento } \\
\text { sustentável da sociedade a partir de uma } \\
\text { perspectiva européia. }\end{array}$ & Estudo teórico & \\
\hline 8 & $\begin{array}{l}\text { Macedo et al. } \\
\qquad(2008)\end{array}$ & $\begin{array}{c}\text { Investigar lugares favoritos, lugares evitados } \\
\text { e afetos envolvidos nessa relação. }\end{array}$ & $\begin{array}{l}\text { Levantamento } \\
\text { de dados }\end{array}$ & Entrevista semiestruturada \\
\hline 9 & $\begin{array}{l}\text { Pinheiro } \\
\text { (1997) }\end{array}$ & $\begin{array}{l}\text { Apresentar um histórico da PA com enfoque } \\
\text { nas implicações teóricas e práticas. }\end{array}$ & Estudo teórico & \\
\hline 10 & Elali (1997) & $\begin{array}{l}\text { Discutir a PA enquanto locus privilegiado na } \\
\text { intersecção entre Psicologia e Arquitetura. }\end{array}$ & Estudo teórico & \\
\hline 11 & $\begin{array}{c}\text { Felippe e } \\
\text { Kuhnen (2012) }\end{array}$ & $\begin{array}{l}\text { Avaliar as práticas de investigação do apego } \\
\text { ao lugar quanto ao modo como se } \\
\text { relacionam às características dos estudos da } \\
\text { PA. }\end{array}$ & Estudo teórico & \\
\hline 12 & $\begin{array}{l}\text { Kuhnen et al. } \\
\qquad(2010)\end{array}$ & $\begin{array}{l}\text { Evidenciar a importância da relação entre a } \\
\text { qualidade dos ambientes e a saúde humana. }\end{array}$ & Estudo teórico & \\
\hline
\end{tabular}


Farias e

16

(2013)

Polli \&

17 Kuhnen (2011)

18

Martinez e

Emmel (2013)

19

Porto (2014)

20 Corrêa e

Bassani (2015)

Zuchiwschi e Fantini (2015) Alencar e
Porciuncula e

Investigar os impactos na vida dos moradores e na cidade de São João del-Rei decorrentes da adesão da Universidade ao

Programa de Apoio a Planos de

Reestruturação e Expansão das

Universidades Federais (REUNI).

Apresentar e discutir a PA desde sua origem até a contemporaneidade.

Investigar de que forma as características sócio-físicas do espaço público intervêm no comportamento de crianças.

Identificar e caracterizar tais lugares, aqui denominados vizinhanças vivas.

Ressaltar as contribuições da teoria de representações sociais na investigação em PA.

Elaborar um checklist para avaliar condições espaciais da residência de idosos.

Descrever as relações proximais e conceituais de educação ambiental e suas articulações com os idosos.

Promover um possível diálogo a partir das contribuições da PA e da Logoterapia.

Identificar e comparar a intenção comportamental de agricultores da região Oeste de Santa Catarina para a conservação e manejo de florestas nativas em seus imóveis rurais.

Retratar o sentimento de comunidade e discutir o conceito de apego ao lugar desenvolvido por Giuliani e as contribuições de Bowlby sobre a teoria do apego.

Discutir a relação de turismo e memórias de lugar.

Discutir a respeito do lugar da alteridade na Freire (2007)
PA, analisando as categorias: apropriação do espaço, identidade de lugar, apego a lugares e mapas afetivos.
Multimétodos
Levantamento de dados

Entrevistas semiestruturadas

Estudo teórico

Multimétodos

Levantamento de dados

Estudo teórico

Multimétodos

Avaliação ambiental, entrevistas, avaliação do mobiliário, medidas antropométricas.

Multimétodos

Entrevistas, História de Vida e diário de campo.

Estudo teórico

Levantamento de dados

Estudo teórico

Estudo teórico
Visitas formais e informais, observações, entrevistas abertas, registro fotográfico, consulta a fontes primárias e secundárias (documentação). 


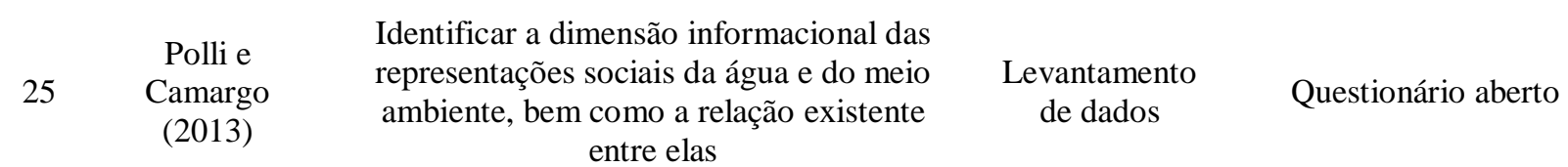
$26 \quad$ Machado et al.

27

Lopez et al. (2012)

28

Salvador et al. (2009)

29

Khoury e Günther
Conhecer a interação criança-natureza nas brincadeiras em dois parques verdes urbanos de Florianópolis (SC).

Multimétodos

Protocolo de observação e utilizada a técnica de mapeamento comportamental centradona-pessoa.

Estudo teórico envelhecimento a partir dos estudos pessoaambiente.

Analisar a associação da prática de atividades físicas no lazer com a percepção do ambiente por idosos.

Multimétodos

Questionário Internacional de Atividades Físicas versão longa, avaliação do ambiente por meio de escala de percepção adaptada do instrumento Neighborhood Environmental Walkability Scale.

Investigar a relação entre ambiente de moradia e duas modalidades de controle percebido, referentes a esforços (2008) empreendidos para adaptar o ambiente às
Multimétodos sociodemográfico, Escala de controle (ECOPSE) próprias necessidades e realizar metas e adaptar-se ao ambiente.

\footnotetext{
* Nota específica: o critério utilizado para a classificação em multimétodos foi identificação do uso de técnicas verbais e não-verbais e/ou análises quantitativo-descritivas e qualitativo-avaliativas.
}

Partindo de uma análise metodológica, dos 14 artigos empíricos que adotaram métodos de levantamento e multimétodos em contextos naturais, nove utilizaram entrevistas como ferramenta de coleta de dados e quatro utilizaram questionários. Houve a utilização de observação direta ou indireta (centrada na pessoa ou no ambiente), levantamento de características do ambiente e da planta baixa, desenho temático, mapeamento comportamental, ambiente fotografado, história de vida, diário de campo e roteiro de observação. A associação de dois ou mais métodos é uma característica descrita por Pinheiro e Günther (2008) como forte e necessária em estudos pessoa-ambiente devido a multiplicidade de disciplinas que estão associadas e devido a própria díade que obriga o pesquisador adotar métodos não somente centrados em um fenômeno, mas na transação entre a pessoa-ambiente.
A partir dos achados, evidenciou-se a forte característica da Psicologia Ambiental acerca de seu caráter interdisciplinar e multidisciplinar em decorrência da variedade de temáticas e públicos abordados (Pinheiro, 1997; Günther et al., 2008; Felippe, 2009; Vlek, 2003). Em relação aos estudos empíricos, evidenciaram-se métodos mais centrados no lugar (Elali, 2003; Farias \& Pinheiro, 2013), ou centrado na pessoa (Khoury \& Günther, 2008; Machado et al., 2016; Polli \& Camargo, 2013; Porciuncula \& Porto, 2014; Salvador et al., 2009), ou na transação dessa díade (Albuquerque, Silva \& Kuhnen, 2018; Araújo \& Santos, 2014; Luz \& Kuhnen, 2013; Macedo et al., 2008; Ros \& Crus, 2011; Zuchiwschi \& Fantini, 2015).

Sob a ótica do lugar, Elali (2003), investigou características físicas em ambientes de educação infantil. Ainda no que concerne à ambientes escolares infantis, Luz e Kuhnen 
(2013) buscaram compreender as características sócio-físicas do ambiente e como estas influenciam o comportamento de crianças. Ainda com o público infantil, embora adotando métodos centrados-na-pessoa, Machado et al. (2016) propuseram um estudo de forma a conhecer as interações entre crianças e natureza em um parque de Florianópolis, utilizando o método observacional. Inseridos ainda em contexto educacional, embora de ensino superior e empregado métodos centrado na transação pessoa-ambiente, Albuquerque, Silva e Kuhnen (2016) investigaram as preferências ambientais de estudantes e elementos físicos restauradores da atenção.

\section{Macedo et al. (2008) também buscaram investigar o fenômeno de preferência de lugar, mas com vistas a perspectiva de idosos e contemplando seus afetos de valência positiva e negativa sobre o lugar. Ainda com o público idoso, Martinez e Emmel (2013) buscaram com seu estudo esboçar um checklist para avaliar as condições espaciais de residências de idosos, para transformá-lo em um roteiro de avaliação. De modo similar à premissa apontada pelo estudo anterior, Khoury e Günhter (2008) investigaram o controle ambiental percebido de idosos sobre sua moradia, utilizando como forma de coleta de dados um roteiro de entrevista e uma escala de controle. Já Porciuncula e Porto (2014) estudaram relações proximais e conceituais de educação ambientais com idosos institucionalizados, utilizando à história de vida como ferramenta de coleta de dados centrado na pessoa. Estudando o fenômeno da percepção ambiental em idosos está Salvador et al. (2009), relacionando-o com as atividades físicas praticadas por este público.}

No que tange aos usuários de lugares com vistas à inter-relação pessoa-ambiente, Zuchiwschi e Fantini (2015) investigaram o fenômeno da intenção comportamental de conservação de agricultores sob florestas nativas; Ros e Cruz (2011) abordaram moradores de uma região para investigar sua história de vida e relação com memórias de lugar; Polli e Camargo (2013) debruçaram-se sobre as representações sociais de homens e mulheres sobre o meio ambiente e a água; Araújo e Santos (2014) se interessaram pelo estudo do impacto da expansão universitária em uma cidade, abordando seus moradores para levantar os prós e contras desse fenômeno ambiental; e Farias e Pinheiro (2013) levantaram aspectos sociais, físicas, culturais, econômicas e ambientais com 10 especialistas em elementos sócio-urbanos para compreender as qualidades de vizinhanças que tornam-as 'vivas'.

No que se refere aos estudos teóricos contemplados neste artigo, também se percebeu uma multiplicidade de temas investigados. Desde a Psicologia Ambiental de um ponto de vista Ecológico (Carneiro \& Bindé, 1997), até uma abordagem mais direcionada à interseção com a Arquitetura (Elali, 1997), com a Logoterapia (Corrêa \& Bassani, 2015), com a Psicologia Comunitária (Lima \& Bomfim, 2009) e de uma relação de PA com o uso da Teoria das Representações Sociais para explicar a díade pessoa-ambiente (Polli \& Kuhnen, 2011). Moser (1998), Pinheiro (1997) e Rivlin (2003) desenvolvem seu estudo teórico com maior ênfase no desenvolvimento, aspectos históricos e epistemológicos da PA. Além disso, ressaltam-se os estudos que abarcam a temática da sustentabilidade (Corrêa \& Bassani, 2015; Vlek, 2003), onde Vlek (2003) discute os dilemas imbricados na qualidade de vida sustentável e fatores resultantes da globalização da sociedade européia, enquanto Corrêa e Bassani (2015) realizam articulações entre PA e Logoterapia para dialogar sobre o cuidado ambiental. Além disso, foram investigados fenômenos como lugares favoritos (Lopez, Felippe, \& Kuhnen, 2012), vinculação afetiva entre pessoa-ambiente (Lima \& Bomfim, 2009), arranjos espaciais (Campos-de-Carvalho, 2003), mobilidade e affordance (Günther, 2003), apego ao lugar (Felippe \& Kuhnen, 2012), assim como esta última temática aliada à outras, como apropriação, territorialidade, privacidade (Kuhnen et al., 2010), assim como o acréscimo 
de identidade de lugar, hospitalidade e mapas afetivos (Alencar \& Freire, 2007).

Nos estudos, apresentou-se uma tendência a abordagens interacionistas e transacionalistas, mas não há um consenso entre os autores. Como supracitado, há estudos que abordam um fenômeno em detrimento de outros, como aqueles que focalizam no modo como à pessoa utiliza o ambiente, assim como as reações psicológicas e físicas que o ambiente causa na pessoa. Ressalta-se que para compreender a relação complexa entre pessoa-ambiente, é necessário que o pesquisador adote uma abordagem transacionalista, para conhecer como se permeia essa inter-relação a partir de uma perspectiva holística, de sistema. As abordagens interacionista e transacionalista também trazem uma marca no que diz respeito ao distanciamento do indivíduo em relação ao meio com o qual ele interage. $\mathrm{Na}$ primeira perspectiva, pessoa e ambiente tendem a formar unidades de análise distintas e, embora tenham o mesmo peso para a investigação (ambas são base para a explicação dos fenômenos psicológicos), comparecem como entidades separadas, uma exercendo influência sobre a outra. No transacionalismo, entretanto, pessoa e ambiente são partes constituintes de um sistema integrado de correlações, cuja compreensão torna-se necessária para o entendimento de quaisquer aspectos da relação humano-ambiental. Aqui, a componente pessoa é investigada, não à parte, mas inserida em seu contexto (Moser, 1998; Pinheiro, 1997; Rivlin, 2003; Valera, 1996).

Diferentes em seus modos de considerar a pessoa e o entorno, as duas perspectivas se desdobram em epistemologias também distintas: realista, no primeiro caso, onde o pesquisador distancia-se do fenômeno para que a realidade produza o conhecimento; e interacionista construtivista, no segundo, onde o conhecimento é parcialmente definido por certas qualidades do observador, tomado como parte do evento. Nessa linha de pensamento, Ittelson, Proshansky, Rivlin, \& Winkel, (2005) e Pinheiro (2003) defendem que o percebedor sempre estará imerso no ambiente, o que em princípio poderá significar: influenciar e ser influenciado pelo contexto da investigação.

\section{Considerações finais}

De modo a responder aos objetivos de pesquisa propostos, identificou-se que o objeto de estudo da PA trata-se da relação pessoaambiente, buscando estudar tal díade como uma relação recíproca, isto é, de mútua influência. Em relação aos métodos e metodologias mais empregados em PA, foi verificado que existem diversas formas de abordar essa relação pessoa-ambiente, com método mais centrado na pessoa ou no lugar, assim como o uso de ambos. A partir dos achados, verificou-se maior amplitude de técnicas em PA e a utilização de mais de uma técnica em conjunto, como o uso de questionários, entrevista com variação da técnica walk around the block, entrevista com variação da técnica ambiente fotografado, mapeamento comportamental, cognitivo e/ou afetivo, análise de vestígios ambientais, autobiografia e construção de maquetes.

No tocante aos aspectos relacionados a ontologia, antropologia e epistemologia, os artigos contemplados nesta pesquisa apontam para uma ontologia é materialista já que existe uma realidade objetiva na qual respondemos por meio de nossa percepção de real. A antropologia transacionalista e agente, que considera o indivíduo como ativo no processo de causar e sofrer transformações diante da realidade que se apresenta. Epistemologia interacionista construtivista, na qual o conhecimento se constrói a partir da interação entre observador e realidade em que se objetiva estudar.

Percebeu-se que os artigos empíricos que abordam os fenômenos utilizando de métodos centrados na pessoa ou no ambiente de modo exclusivo adotam uma perspectiva interacionista da relação pessoa-ambiente. Há uma percepção nesses estudos de que um influencia o outro, mas que ambos não estão equivalentes em um mesmo sistema. Já os estudos que utilizam multimétodos, com vistas 
aos dois fenômenos imbricados em uma relação complexa, adotam a perspectiva que seria a ideal em Psicologia Ambiental: transacionalista. Compreender o homem e o ambiente inseridos em um sistema de mútua influência e transformação é palco para entender os fenômenos que se propõe a investigar, sejam eles psicológicos da pessoa e/ou ambientais do espaço físico. Portanto, foi verificado que não existe um consenso entre os autores no que tange a perspectiva, mas indica-se as futuras pesquisas que adotem a perspectiva transacionalista, considerando o indivíduo sempre no ambiente, não a parte dele.

Além disso, analisou-se que as pesquisas empíricas têm adotado a abordagem multimétodos, principalmente utilizando como instrumentos de coleta de dados as entrevistas e questionários, atribuindo a PA essa característica como sendo crucial e quase determinante. Contudo, destaca-se que a abordagem multimétodos não é própria e exclusivamente utilizada pela PA, pois são empregados em estudos de diversas áreas do conhecimento. Entretanto, como as pesquisas demonstram a dificuldade em pesquisar dois fenômenos e a relação entre eles, evidencia-se a necessidade de utilizar metodologias diversificadas para possibilitar maior aprofundamento e alcançar resultados fidedignos.

Por fim, outro aspecto que apresentouse com nitidez nos achados diz respeito a multidisciplinaridade em PA, principalmente devido ao cruzamento de profissões, desde aquelas que possuem como especialidade o ambiente físico (Arquitetura, Urbanismo, Paisagismo, Geografia) aquelas que focalizam aspectos psicológicos (Psicologia), sociais (Sociologia, Antropologia) e aspectos biológicos e orgânicos (Biologia, Medicina). Embora seja um trabalho enriquecedor a união de disciplinas e profissões para estudar processos psicológicos e físicos decorrentes dessa díade, há incumbido nessa ação multidisciplinar diversas dificuldades que permeiam o trabalho em equipe. Dessa forma, os pesquisadores necessitam inteirar-se para unir conhecimento, sem focalizar um ou outro fenômeno conforme sua especialidade (pessoa ou ambiente), mas acrescentar conhecimento ao estudo de forma funcional e interdisciplinar efetivamente.

\section{Referências}

Albuquerque, C. A. D., \& Albuquerque, U. P. D. (2005). Local perceptions towards biological conservation in the community of vila velha, Pernambuco, Brazil.

Interciencia, 30(8), 460-465. Recuperado de

https://medioambienteycultura.files.wordpr ess.com/2014/09/local-perceptionstowards-biological-conservation.pdf.

Albuquerque, D. S., Silva, D. S., \& Kuhnen,

A. (2016). Preferências Ambientais e Possibilidades de Restauro Psicológico em Campi Universitários. Psicologia: Ciência e Profissão, 36(4), 893-906. doi: 10.1590/1982-3703002972015

Alencar, H. F., \& Freire, J. C. (2007). O lugar da alteridade na psicologia ambiental. Revista Mal-Estar e Subjetividade, 7(2), 305-328. Recuperado de http://pepsic.bvsalud.org/scielo.php?script= sci_arttext\&pid=S1518$61482007000200005 \& \operatorname{lng}=p t \& t \operatorname{lng}=p t$. Araújo, C. B. de, \& Santos, L. M. M. (2014). Impactos da expansão universitária para moradores de São João Del-Rei. Psicologia \& Sociedade, 26(2), 420-429. doi: 10.1590/S0102-71822014000200018

Beyea, S. C., \& Nicoll, L. H. (1998). Writing in integrative review. AORN Journal, 67, 877-880. doi: 10.1016/S00012092(06)62653-7

Campos-de-Carvalho, M. I. (2003). Pesquisas contextuais e seus desafios: uma contribuição a partir de investigações sobre arranjos espaciais em creches. Estudos de Psicologia (Natal), 8(2), 289-297. doi: $\underline{10.1590 / S 1413-294 X 2003000200011}$ 
Carneiro, C., \& Bindé, P. J. (1997). A Psicologia Ecológica e o estudo dos acontecimentos da vida diária. Estudos de Psicologia (Natal), 2(2), 363-376. doi: $\underline{\text { 10.1590/S1413-294X1997000200010 }}$

Corral-Verdugo, V. (2005). Psicologia Ambiental: objeto, "realidades" sóciofísicas e visões culturais de interações ambiente-comportamento. Psicologia USP, 16(1/2), 71-87. Recuperado de http://pepsic.bvsalud.org/pdf/psicousp/v16n 1-2/24645.pdf

Corrêa, D. A., \& Bassani, M. A. (2015). Cuidado ambiental e responsabilidade: possível diálogo entre Psicologia Ambiental e logoterapia. Psicologia em Estudo, 20(4), 639-649. Recuperado de http://www.redalyc.org/articulo.oa?id=2871 45780012

Elali, G. A. (1997). Psicologia e Arquitetura: em busca do locus interdisciplinar. Estudos de Psicologia (Natal), 2(2), 349-362. doi: 10.1590/S1413-294X1997000200009

Elali, G. A. (2003). O ambiente da escola - o ambiente na escola: uma discussão sobre a relação escola-natureza em educação infantil. Estudos de Psicologia (Natal), 8(2), 309-319. doi: 10.1590/S1413294X2003000200013

Elali, G. A. (2007). Imagem sócio-ambiental de áreas urbanas: um estudo na Ribeira, Natal-RN-Brasil. Psicologia para a América Latina (10), 0-0. Recuperado de http://pepsic.bvsalud.org/scielo.php?script= sci_arttext\&pid=S1870350X2007000200006\&lng=pt\&tlng=pt

Farias, T. M., \& Pinheiro, J. Q. (2013). Vivendo a vizinhança: interfaces pessoaambiente na produção de vizinhanças "Vivas". Psicologia em Estudo, 18(1), 2736. doi:10.1590/S141373722013000100004

Felippe, M. L. (2009). Ambiente pessoal: o papel da personalização na construção de espaços saudáveis. In K. E. Takase, \& R. M. Cruz (Eds.), Interações pessoaambiente e saúde. São Paulo, SP: Casa do Psicólogo.

Felippe, M. L., \& Kuhnen, A. (2012). O apego ao lugar no contexto dos estudos pessoa- ambiente: práticas de pesquisa. Estudos de Psicologia (Campinas), 29(4), 609-617. doi:10.1590/S0103-166X2012000400015

Günther, H. (2003). Mobilidade e affordance como cerne dos Estudos Pessoa-Ambiente. Estudos de Psicologia (Natal), 8(2), 273280. doi: 10.1590/S1413294X2003000200009

Günther, H., Elali, G. A., \& Pinheiro, J. Q. (2008). A abordagem multimétodos em estudos pessoa-ambiente: características, definições e implicações. In J. d. Q. Pinheiro \& H. Günther (Eds.), Métodos de pesquisa nos estudos pessoa-ambiente. São Paulo: Casa do Psicólogo.

Ittelson, W. H., Proshansky, H. M., Rivlin, L. G., \& Winkel, G. H. (2005). Homem ambiental. Série: Textos de Psicologia Ambiental, 14.

Khoury, H. T. T., \& Günther, I A. (2008). Ambiente de moradia e controle primário em idosos. Paidéia, 18(39), 53-60.

Recuperado de http://www.redalyc.org/articulo.oa?id=3054 23760006

Kruse, L. (2005). Compreendendo o ambiente em Psicologia Ambiental. Psicologia USP, 16(1/2), 41-46. Recuperado em http://pepsic.bvsalud.org/pdf/psicousp/v16n 1-2/24642.pdf

Kuhnen, A., Felippe, M. L., Luft, C. Di B., \& Faria, J. G. de. (2010). A importância da organização dos ambientes para a saúde humana. Psicologia \& Sociedade, 22(3), 538-547. doi: 10.1590/S010271822010000300014

Lima, D. M., \& Bomfim, Z. A. C. (2009). Vinculação afetiva pessoa-ambiente: diálogos na psicologia comunitária e psicologia ambiental. Psico, 40(4), 491497. Recuperado de https://dialnet.unirioja.es/servlet/articulo?co digo $=5161393$

Lopez, M., Felippe, M. L., \& Kuhnen, A. (2012). Lugares favoritos no envelhecimento: Explorando estudos e conceitos. Psicologia Argumento, 30(71), 639-649. doi:

$\underline{10.7213 / \text { psicolargum.v30i71.20137 }}$ 
Luz, G. M., \& Kuhnen, A. (2013). O uso dos espaços urbanos pelas crianças: explorando o comportamento do brincar em praças públicas. Psicologia: Reflexão e Crítica, 26(3), 552-560. doi: 10.1590/S010279722013000300015

Macedo, D., Oliveira, C. V., Günther, I. A., Alves, S. M., \& Nóbrega, T. S. (2008). O lugar do afeto, o afeto pelo lugar: o que dizem os idosos?. Psicologia: Teoria e Pesquisa, 24(4), 441-449. doi: 10.1590/S0102-37722008000400007

Machado, Y. S., Schubert, P. M. P., Albuquerque, D. da S., \& Kuhnen, A. (2016). Brincadeiras infantis e natureza: investigação da interação criança-natureza em parques verdes urbanos. Temas em Psicologia, 24(2), 655-667. doi:10.9788/TP2016.2-14Pt

Marconi, M. A., \& Lakatos, E. M. (2017). Fundamentos de metodologia científica $\left(8^{\mathrm{a}}\right.$ Ed.). São Paulo, SP: Atlas.

Martinez, L. B. A., \& Emmel, M. L. G. (2013). Elaboração de um roteiro para avaliação do ambiente e do mobiliário no domicílio de idosos. Revista de Terapia Ocupacional da Universidade de São Paulo, 24(1), 18-27. doi: 10.11606/issn.2238-6149.v24i1p18-27

Moser, G. (1998). Psicologia Ambiental. Estudos de Psicologia, 3(1), 121-130. Recuperado de https://pt.scribd.com/document/213512802/ 1998-Art-Psicologia-Ambiental-Moser

Pinheiro, J. Q. (1997). Psicologia Ambiental: a busca de um ambiente melhor. Estudos de Psicologia (Natal), 2(2), 377-398. doi: 10.1590/S1413-294X1997000200011

Pol, E. (1993). Environmental Psychology in Europe: from Architectural Psychology to Green Psychology. Aldershot: Avebury.

Pol, E. (2006). Blueprints for a history of environmental psychology (I): From first birth to American transition. Medio Ambiente y Comportamiento Humano, 7(2), 95-113.

Pol, E. (2007). Blueprints for a history of environmental psychology (II): From architectural psychology to the challenge of sustainability. Medio Ambiente y Comportamiento Humano, 8(1/2), 1-28.

Polli, G. M., \& Kuhnen, A. (2011).

Possibilidades de uso da teoria das representações sociais para os estudos pessoa-ambiente. Estudos de Psicologia (Natal), 16(1), 57-64. doi: 10.1590/S1413294X2011000100008

Polli, G. M., \& Camargo, B. V. (2013). Meio ambiente e água sob a perspectiva da teoria das representações sociais. Psicologia Ciência e Profissão, 33(2), 256-271.

Recuperado de http://www.redalyc.org/articulo.oa?id=2820 27993002

Porciuncula, A. D. S., \& Porto, I. (2014). Envelhecimento, meio ambiente e educação ambiental. Estud. interdiscip. envelhec, 19(2), 453-470. Recuperado de http://www.seer.ufrgs.br/RevEnvelhecer/art icle/viewFile/39360/32758

Rivlin, L. G. (2003). Olhando o passado e o futuro: revendo pressupostos sobre as interrelações pessoa-ambiente. Estudos de Psicologia, 8(2), 215-220. doi:10.1590/S1413-294X2003000200003

Ros, J. P., \& Cruz, W. F. O. (2011). Memórias do lugar: o turismo na ilha do campeche. Psicologia \& Sociedade, 23(3), 625-633. doi: 10.1590/S0102-71822011000300021

Salvador, E. P., Florindo, A. A., Reis, R. S., \& Costa, E. F. (2009). Percepção do ambiente e prática de atividade física no lazer entre idosos. Revista de Saúde Pública, 43(6), 972-980. doi: 10.1590/S0034$\underline{89102009005000082}$

Tassara, E. T. O., \& Rabinovich, E. P. (2003). Perspectivas da Psicologia Ambiental. Estudos de Psicologia, 8(2), 339-340. doi:10.1590/S1413-294X2003000200018

Tuan, Y.-F. (1983). Espaço e lugar: a perspectiva da experiência. São Paulo: Difel.

Valera, S. (1996). Psicología Ambiental: bases teóricas y epistemológicas. In L. Iñiguez, \& E. Pol (Eds.), Cognición, representación y apropiación del espacio (pp. 1-14). Barcelona: Universidad de Barcelona Publicacions. 
Vlek, C. (2003). Globalização, dilemas dos comuns e qualidade de vida sustentável: do que precisamos, o que podemos fazer, o que podemos conseguir?. Estudos de Psicologia (Natal), 8(2), 221-234. doi: 10.1590/S1413-294X2003000200004.

Wiesenfeld, E. (2005). A Psicologia Ambiental e as diversas realidades humanas. Psicologia USP, 16(1/2), 53-69. Recuperado de http://www.scielo.br/pdf/pusp/v16n12/24644.pdf.

Zuchiwschi, E., \& Fantini, A. C. (2015). Intenção comportamental de agricultores do Oeste de Santa Catarina para a conservação de florestas nativas. Estudos de Psicologia, 20(1), 61-71. Recuperado de http://decubacubawww.redalyc.org/articulo. oa?id=26142192008.

\section{Dados sobre os autores:}

- Maísa Hodecker: Mestranda em Psicologia pela Universidade Federal de Santa Catarina. Integrante do Laboratório de Psicologia Ambiental (LAPAM/UFSC). Formou-se em Psicologia pelo Centro Universitário de Brusque - UNIFEBE. Tem experiência em Gestão em Emergências e Desastres, Psicologia Ambiental e Promoção e Prevenção da Saúde. No decorrer de sua trajetória acadêmica, atuou na Clínica Escola e Serviços de Psicologia (CESP/UNIFEBE) e na realização de estágio obrigatório no Corpo de Bombeiros da cidade de Brusque/SC (2016). Foi auxiliar de pesquisa do Art. 170 no Centro Universitário de Brusque UNIFEBE. É bolsista CAPES-DS.

- Igor Schütz dos Santos: Psicólogo graduado pela Universidade Federal de Santa Catarina (UFSC). Mestrando em Psicologia na UFSC, vinculado ao Laboratório de Psicologia Ambiental (LAPAM). Possui especialização em Gestão de Recursos Humanos e Meio Ambiente pela Universidade Cândido Mendes (UCAM) e formação em psicanálise pela Maiêutica Florianópolis. Psicólogo da Companhia Catarinense de Águas e Saneamento (CASAN), atuando na Gerência Universidade Corporativa.

- Maíra Longhinotti Felippe: Possui graduação em Arquitetura e Urbanismo (Universidade Federal de Santa Catarina, 2001), mestrado em Psicologia (Universidade Federal de Santa Catarina, 2010) e doutorado em Tecnologia da Arquitetura (Università degli Studi di Ferrara, Itália, 2015). Atualmente é Professora Substituta na Universidade Federal de Santa Catarina e Pesquisadora em Pós-Doutorado no Laboratório de Psicologia Ambiental - LAPAM, no Departamento de Psicologia da mesma instituição. Tem experiência no campo de conhecimento da Expressão Gráfica e dos Estudos Pessoa-Ambiente, Psicologia Ambiental, especificamente nos seguintes temas: cuidado ambiental, apego ao lugar, linguagem e significado ambiental, ambientes restauradores, Arquitetura Escolar e Hospitalar. Dedica-se ao estudo do modo como ambientes para o cuidado da saúde, como hospitais, podem se tornar restauradores a partir de suas características físicas e da relação que as pessoas estabelecem com o lugar.

- Daniela Ribeiro Schneider: Possui graduação em Psicologia pela Universidade Federal de Santa Catarina (1987), mestrado em Educação pela Universidade Federal de Santa Catarina (1993), doutorado em Psicologia (Psicologia Clínica) pela Pontifícia Universidade Católica de São Paulo (2002) e Pós-Doutorado pela Universidade de Valencia - Espanha (2012). Atualmente é Prof. Associado IV da Universidade Federal de Santa Catarina. Tem experiência na área de Psicologia, com ênfase em Tratamento e Prevenção Psicológica, atuando principalmente nos seguintes temas: problemas relacionados ao uso de álcool e drogas, programas de prevenção e serviços de saúde que atendem usuários de drogas, atenção 
psicossocial, história da psicologia, Jean-Paul Sartre, psicologia existencialista. Coordenadora do Grupo de Pesquisa do CNPQ "Clínica da Atenção Psicossocial e Uso de Álcool e Outras Drogas". Coordenadora do Nùcleo de Pesquisa em Clínica da Atenção Psicossocial PSICLIN/UFSC. Bolsista produtividade em pesquisa 2 pelo CNPQ.

- Ariane Kuhnen: Psicóloga e docente. Psicóloga, Mestre em Sociologia Política, Doutora em Ciências Humanas, Professora do Departamento de Psicologia e do Programa de PósGraduação em Psicologia da Universidade Federal de Santa Catarina. Coordenadora do Laboratório de Psicologia Ambiental-LAPAM. Exerce atividades de ensino, pesquisa extensão na área de Psicologia, com ênfase em psicologia educacional e psicologia ambiental. Coordenadora do Grupo de Trabalho de Psicologia Ambiental da ANPEPP - Associação Nacional de Pesquisa e Pós-Graduação em Psicologia. Coordenadora dos Acordos de Cooperação entre UFSC e Université de Nîmes (France) e; entre UFSC e University of California-UCDavis (USA). 